\title{
The Role of Polarization in The Multiple Reflections Modeling
}

\author{
Alexander Y. Basov \\ Light Engineering \\ Department Moscow \\ Power Engineering \\ Institute, Moscow \\ Russia \\ BasovAlY@mpei.ru
}

\author{
Vladimir P. Budak \\ Light Engineering \\ Department Moscow \\ Power Engineering \\ Institute, Moscow \\ Russia \\ BudakVP@gmail.com
}

\author{
Anton V. Grimailo \\ Light Engineering \\ Department Moscow \\ Power Engineering \\ Institute, Moscow \\ Russia \\ GrimailoAV@gmail.com
}

\begin{abstract}
This article shows a new mathematical model for calculation of multiple reflections based on the Stokes vector and Mueller matrices. The global illumination equation and local estimations method were generalized on the polarization case. The results of the calculation of multiple reflections using the local estimations method show a difference of more than $30 \%$ between the standard calculation and the polarization-accounting one. A way to describe the surface reflection with polarization account is proposed.
\end{abstract}

\section{Keywords}

Polarization, global illumination equation, local estimations, Stokes vector, Mueller matrix, Monte-Carlo.

\section{INTRODUCTION}

In recent years, visualization of the light distribution obtained by using a lighting device has become an integral part of the design of lighting systems. Thus, the calculation of multiple reflections has become exceedingly important.

Traditionally, the state of light polarization is not considered in the calculations. Similarly, lighting modeling software (e.g. the industry standard software DIALux and Relux) do not consider the influence of the light polarization in the calculation of light distribution.

This neglection is acceptable when we work with diffusely reflecting surfaces and a small number of re-reflections. On the contrary, the influence of light polarization must be considered by surfaces with a significant specular part. The very first reflection changes the state of light polarization (even if the light has been depolarized totally before the action) and this fact will affect all the following processes of light distribution.

It is evident that after a series of reflections the light becomes depolarized again. However, the effect of the light polarization on the quantitative results of the

Permission to make digital or hard copies of all or part of this work for personal or classroom use is granted without fee provided that copies are not made or distributed for profit or commercial advantage and that copies bear this notice and the full citation on the first page. To copy otherwise, or republish, to post on servers or to redistribute to lists, requires prior specific permission and/or a fee. calculation remains unknown. By current estimates [Mishchenko et al. 1994], the difference between results of the standard calculation and the calculation considering the polarization may reach more than $20 \%$. This difference may be even more significant if we use the polarization-based model of reflecting surface which considers the light scattering in a material volume.

To date, a series of proceedings devoted to the polarization account in visualization problems have been published (e.g. [Mojzik et al. 2016]). They show that polarization state accounting leads to quite significant changes in the pictures obtained during visualization. However, despite of the abundance of research of such kind, the question of the light polarization influence on the quantitative characteristics of the light distribution has not been considered yet. At the same time, it is these characteristics that primarily interest light planners when they solve practical problems.

Thus, we decided to study the influence of the light polarization on the quantitative result of the calculation of multiple reflections. To achieve this goal, we need to solve the following tasks.

1. Pass from the wave description of the light to the ray approximation as the latter is more in nature of the phenomena considered.

2. Bring the global illumination equation for the Stokes vector to the volume integration. Existing equation based on surface integration [Mojzik et al. 2016] does not allow constructing mathematically rigorous ray-based algorithms. 
3. Calculate multiple reflections using local estimations of the Monte-Carlo method (as they appear to be the most efficient for solving the problem) and evaluate the influence of the light polarization account on the quantitative result.

We are also confident there is a need for a mathematical model of reflecting surface describing the light reflection not only from the face of material but from the volume of material as well. In this case, the role of the light polarization is highly significant too.

\section{MATHEMATICAL MODEL OF MULTIPLE REFLECTIONS \\ WITH POLARIZATION ACCOUNT}

Description of polarization in the electromagnetic field theory using ellipses is completely unknown to the description of radiation in ray optics which is based on the reaction of the optical radiation receiver. The most suitable way to develop the mathematical model with polarization-accounting parameters would be to use the Stokes vector (for describing the ray parameters) and $4 \times 4$ Mueller matrices [Mueller H. 1943] (for describing the parameters of surface reflection).

The Stokes vector $\mathbf{L}$ components are determined by the quadratic receiver reactions $J_{i}$ as

$$
\begin{gathered}
L_{0}=2 J_{0}, \quad L_{1}=2\left(J_{0}-J_{1}\right), \\
L_{2}=2\left(J_{2}-J_{0}\right), \quad L_{3}=2\left(J_{3}-J_{0}\right),
\end{gathered}
$$

where specific polarization filters differ $J_{i}, i \in \overline{0,3}$ : $J_{0}$ is for the neutral filter with the transmittance $\tau=0.5 ; \quad J_{1}$ is for the reference plane (the "system of readout") defining analyzer; $J_{2}$ is for the analyzer with the axis at the angle $45^{\circ}$ to the reference plane; $J_{3}$ is for complex filter of quarter-wave plate and analyzer at the angle $45^{\circ}$ to the reference plane.

The result of the interaction of the surface and the ray described with the Stokes vector can be written as:

$$
\mathbf{L}(\mathbf{r}, \hat{\mathbf{l}})=\vec{\rho}\left(\mathbf{r}, \hat{\mathbf{l}}, \hat{\mathbf{I}}^{\prime}\right) \mathbf{L}(\mathbf{r}, \hat{\mathbf{l}})
$$

where $\mathbf{L}\left(\mathbf{r}, \hat{\mathbf{I}}^{\prime}\right)$ and $\mathbf{L}(\mathbf{r}, \hat{\mathbf{l}})$ are the radiances before and after the interaction respectively; $\vec{\rho}\left(\mathbf{r}, \hat{\mathbf{l}}, \hat{\mathbf{l}}^{\prime}\right)$ is the Mueller matrix of the surface (we consider in this article the reflection only). Expression (2) is correct when the reference planes of the incident are the same as the reflected rays. In general case, we must account the rotation of the reference system with a special matrix. The matrix which is the multiplication of each transformation of the Mueller matrix provides the successive transformations result.

Note that hereinafter we use the following notation: a - column vector; $\overline{\mathbf{a}}$ - row vector; $\hat{\mathbf{a}}$ - unit column vector; $\overrightarrow{\mathbf{a}}$ - matrix.

The questions of simulation of lighting systems and visualization of $3 \mathrm{D}$ scenes in computer graphics are based on finding the solution of the global illumination equation [Kajiya J. T. 1986]

$$
\begin{gathered}
L(\mathbf{r}, \hat{\mathbf{l}})=L_{0}(\mathbf{r}, \hat{\mathbf{l}})+ \\
+\frac{1}{\pi} \int L\left(\mathbf{r}, \hat{\mathbf{l}}^{\prime}\right) \sigma\left(\mathbf{r}, \hat{\mathbf{l}}, \hat{\mathbf{l}}^{\prime}\right) F\left(\mathbf{r}, \mathbf{r}^{\prime}\right) \Theta\left(\mathbf{r}, \mathbf{r}^{\prime}\right) d^{2} \mathbf{r}^{\prime}, \\
F\left(\mathbf{r}, \mathbf{r}^{\prime}\right)=\frac{\left|\left(\hat{\mathbf{N}}, \hat{\mathbf{l}}^{\prime}\right)\left(\hat{\mathbf{N}}^{\prime}, \hat{\mathbf{l}}^{\prime}\right)\right|}{\left(\mathbf{r}-\mathbf{r}^{\prime}\right)^{2}}=\frac{\left|\left(\hat{\mathbf{N}}, \mathbf{r}-\mathbf{r}^{\prime}\right)\left(\hat{\mathbf{N}}^{\prime}, \mathbf{r}-\mathbf{r}^{\prime}\right)\right|}{\left(\mathbf{r}-\mathbf{r}^{\prime}\right)^{4}},
\end{gathered}
$$

where $L(\mathbf{r}, \hat{\mathbf{l}})$ is the radiance at the point $\mathbf{r}$ in the direction $\hat{\mathbf{l}}, \sigma\left(\mathbf{r}, \hat{\mathbf{l}}_{,} \hat{\mathbf{l}}^{\prime}\right)$ is used for the bidirectional scattering distribution function (reflection or transmission), $L_{0}(\mathbf{r}, \hat{\mathbf{l}})$ is the direct radiation radiance near the sources, $\hat{\mathbf{N}}$ is the scene surface normal vector, $\Theta\left(\mathbf{r}, \mathbf{r}^{\prime}\right)$ is the function of the surface element $d^{2} \mathbf{r}^{\prime}$ visibility from the point $\mathbf{r}$.

Thus, guiding the considerations used in derivation (3) one can obtain an analogous equation for the Stokes vector:

$$
\begin{gathered}
\mathbf{L}(\mathbf{r}, \hat{\mathbf{l}})=\mathbf{L}_{0}(\mathbf{r}, \hat{\mathbf{l}})+ \\
+\frac{1}{\pi} \oint \overrightarrow{\mathrm{R}}\left(\hat{\mathbf{l}}^{\prime} \times \hat{\mathbf{l}} \rightarrow \hat{\mathbf{N}} \times \hat{\mathbf{l}}\right) \vec{\rho}\left(\mathbf{r}, \hat{\mathbf{l}}, \hat{\mathbf{l}}^{\prime}\right) \times \\
\times \overrightarrow{\mathrm{R}}\left(\hat{\mathbf{N}} \times \hat{\mathbf{l}}^{\prime} \rightarrow \hat{\mathbf{l}}^{\prime} \times \hat{\mathbf{l}}\right) \mathbf{L}\left(\mathbf{r}, \hat{\mathbf{l}}^{\prime}\right) F\left(\mathbf{r}, \mathbf{r}^{\prime}\right) \Theta\left(\mathbf{r}, \mathbf{r}^{\prime}\right) d^{2} \mathbf{r}^{\prime},
\end{gathered}
$$

where $\mathbf{L}(\mathbf{r}, \hat{\mathbf{l}})$ is the Stokes vector; $\overrightarrow{\mathbf{R}}\left(\hat{\mathbf{I}}^{\prime} \times \hat{\mathbf{I}} \rightarrow \hat{\mathbf{N}} \times \hat{\mathbf{I}}\right)$ is the reference plane rotation matrix; $\vec{\rho}\left(\mathbf{r}, \hat{\mathbf{l}}, \hat{\mathbf{I}}^{\prime}\right)$ is the Mueller matrix of reflectance.

In general, the global lighting equation has no analytical solution. For this reason, the numerical methods are used mainly for solving it. The most popular approach is to use the Monte-Carlo Methods, as they have shown to be efficient comparing to other methods.

The algorithm of calculation of lighting systems and visualization of 3D scenes based on local estimations of the Monte-Carlo Method [Kalos M. 1963] seems to be the most advanced in its class. This approach enables to speed up the calculations by $80-90$ times compared to direct modeling [Budak et al. 2012]. This is especially important for polarizationaccounting calculations as they require much more operations on each step of the algorithm.

The general meaning of the transition to the local estimations is the transformation of the surface integration in (4) into the integration over the volume by using $\delta$-function. This enables the development of a ray-based modeling algorithm. 
In order to enable the transformation we need to account that $\mathbf{r}^{\prime}$ and $\hat{\mathbf{l}}^{\prime}$ are connected by the following expression:

$$
\hat{\mathbf{l}}^{\prime}=\frac{\mathbf{r}-\mathbf{r}^{\prime}}{\left|\mathbf{r}-\mathbf{r}^{\prime}\right|} .
$$

Then the equation (4) takes the form:

$$
\begin{gathered}
\mathbf{L}(\mathbf{r}, \hat{\mathbf{l}})=\mathbf{L}_{0}(\mathbf{r}, \hat{\mathbf{l}})+ \\
+\frac{1}{\pi} \int \overrightarrow{\mathrm{R}}\left(\hat{\mathbf{l}}^{\prime} \times \hat{\mathbf{l}} \rightarrow \hat{\mathbf{N}} \times \hat{\mathbf{l}}\right) \vec{\rho}\left(\mathbf{r}, \hat{\mathbf{l}}, \hat{\mathbf{l}}^{\prime}\right) \overrightarrow{\mathrm{R}}\left(\hat{\mathbf{N}} \times \hat{\mathbf{l}}^{\prime} \rightarrow \hat{\mathbf{l}}^{\prime} \times \hat{\mathbf{l}}\right) \times \\
\times \delta\left(\hat{\mathbf{l}}-\hat{\mathbf{l}}^{\prime}\right) \mathbf{L}\left(\mathbf{r}, \hat{\mathbf{l}}^{\prime}\right) F\left(\mathbf{r}, \mathbf{r}^{\prime}\right) \Theta\left(\mathbf{r}, \mathbf{r}^{\prime}\right) d^{3} \mathbf{r}^{\prime} .
\end{gathered}
$$

Now, one can show the integral in (6) as a Neumann series and after some transformations [Marchuk G. I. 1980] get an expression for local estimation of Stokes vector at the point $\mathbf{r}$ :

$$
\begin{aligned}
& \mathbf{I}_{\varphi}=\mathrm{M} \sum_{n=0}^{\infty} \vec{k}\left(\mathbf{r}, \mathbf{r}^{\prime}\right) \mathbf{Q}_{n}, \\
& \vec{k}\left(\mathbf{r}, \mathbf{r}^{\prime}\right)=\overrightarrow{\mathrm{R}}\left(\hat{\mathbf{l}}^{\prime} \times \hat{\mathbf{l}} \rightarrow \hat{\mathbf{N}} \times \hat{\mathbf{l}}\right) \vec{\rho}\left(\mathbf{r}, \hat{\mathbf{l}}, \hat{\mathbf{l}}^{\prime}\right) \overrightarrow{\mathrm{R}}\left(\hat{\mathbf{N}} \times \hat{\mathbf{l}}^{\prime} \rightarrow \hat{\mathbf{l}}^{\prime} \times \hat{\mathbf{l}}\right) \times \\
& \times F\left(\mathbf{r}, \mathbf{r}^{\prime}\right) \Theta\left(\mathbf{r}, \mathbf{r}^{\prime}\right),
\end{aligned}
$$

where $\mathbf{Q}_{n}$ is the vector weight of the ray, which components correspond to those of the Stokes vector.

\section{THE MODEL IMPLEMENTATION AND OBTAINED RESULTS}

The mathematical model described above was implemented using the numerical-computing environment MATLAB. In order to make the algorithm simpler and reduce the calculation time, the authors decided to assume the diffuse distribution of the rays reflected from the surface. This way enables to estimate the first approximation of the influence of polarization account in the multiple reflection calculations.

A $1 \times 1 \times 1$ cube "room" was chosen as a modeling scene. There are points of illuminance estimation on the "floor" and a Lambertian luminous disc (diameter 0.1 ) in the center of the "ceiling" (Figure 1).

The sum of two matrices was used as a reflection matrix when calculating:

$$
\vec{\rho}=a \vec{\rho}_{F}+(1-a) \vec{\rho}_{L},
$$

where $\vec{\rho}_{F}$ is Fresnel reflection (Mueller) matrix; $\vec{\rho}_{L}$ is Lambertian reflection matrix; $a$ is Fresnel part in reflection $(0<a<1)$. Matrix $\vec{\rho}_{L}$ is a zero-matrix with an only non-zero element $\rho_{L 11}$ for the reflection coefficient.

The model surfaces have the following parameters: reflection coefficient -0.5 ; refractive index -1.5 ; $a$ changes from 1 to 0 . Figure 2 demonstrates the results normalized relative to the number of rays emitted from the disc.

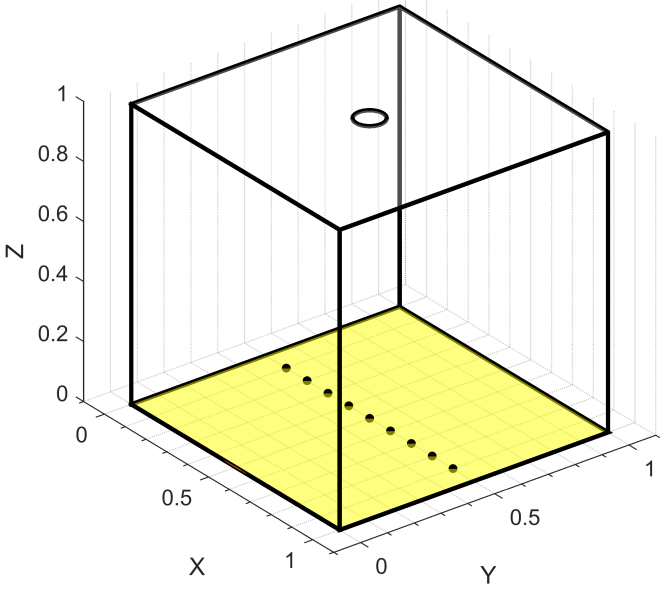

Figure 1. The modeling scene.

One can see, that already at $a=0.2$ the difference between the values of illuminance is about $10 \%$ and at $a=0.6$ more than $20 \%$.

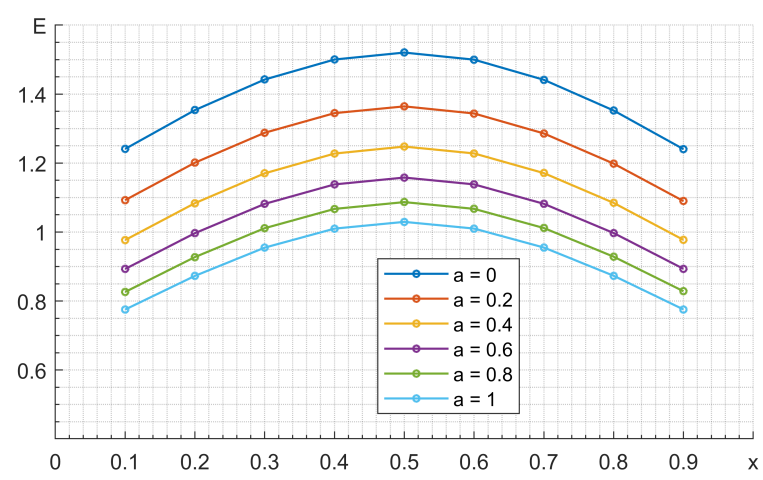

Figure 2. The results of the multiple reflections modeling (relative units).

\section{FURTHER DEVELOPMENT OF THE MODEL}

When calculating multiple reflections, we accept the assumption that the reflected ray diffuse distribution in order to account the light reflection not only from the material surface but also from the material volume. The light penetrates the near-surface layers of the material where the light scattering by the material particles occurs. Then a certain fraction of the initial luminous flux re-enters the surrounding space. At this point, the role of the light polarization is highly significant too.

The light scattering by the material particles in the reflecting layer is inherently similar to the radiative transfer in turbid media. Therefore, at the first stage of model development, we are going to represent the reflecting surface as a scattering layer with diffusely reflecting plane at the bottom and randomly rough Fresnel boundary above. 
In the general case, the scattering media is characterized by matrix scatter coefficient $\vec{\sigma}$, matrix absorption coefficient $\vec{\kappa}$ and matrix extinction coefficient $\vec{\varepsilon}=\vec{\kappa}+\vec{\sigma}$. Neglection of dichroism, birefringence and similar effects which are inherent only for several materials enables the transition to scalar analogs of matrix coefficients $\varepsilon=\kappa+\sigma$.

Thus, for modeling of surface reflection with the assumptions above we need to solve the boundary value problem for the vector radiative transfer equation. Consider the plane-parallel layer with the diffuse bottom. The layer is irradiated by the plane monodirectional source with a random polarization state. Then one can write the problem as

$\left\{\begin{array}{l}\mu \frac{\partial}{\partial \tau} \mathbf{L}(\tau, \hat{\mathbf{l}})+\mathbf{L}(\tau, \hat{\mathbf{l}})=\frac{\Lambda}{4 \pi} \oint \overrightarrow{\mathbf{R}}\left(\hat{\mathbf{l}} \times \hat{\mathbf{l}}^{\prime} \rightarrow \hat{\mathbf{N}} \times \hat{\mathbf{l}}\right) \times \\ \times \vec{x}\left(\tau, \hat{\mathbf{l}}, \hat{\mathbf{l}}^{\prime}\right) \overrightarrow{\mathrm{R}}\left(\hat{\mathbf{N}} \times \hat{\mathbf{l}}^{\prime} \rightarrow \hat{\mathbf{l}} \times \hat{\mathbf{l}}^{\prime}\right) \mathbf{L}(\tau, \hat{\mathbf{l}}) d \hat{\mathbf{l}}^{\prime} ; \\ \mathbf{L}(0, \mu>0, \varphi)=\mathbf{L} \delta\left(\hat{\mathbf{l}}-\hat{\mathbf{l}}^{\prime}\right) ; \\ \mathbf{L}(\tau, \mu \leq 0, \varphi)=\left[\begin{array}{llll}\rho E / \pi & 0 & 0 & 0\end{array}\right]^{T},\end{array}\right.$

where $\mu=\cos \theta ; \quad \tau=\int_{z_{0}}^{z_{1}} \varepsilon(z) d z$ is the optical track thickness in the section $\left[z_{0}, z_{1}\right] ; \Lambda=\sigma / \varepsilon$ is the single scatter albedo; $\vec{x}\left(\tau, \hat{\mathbf{l}}, \hat{\mathbf{l}}^{\prime}\right)$ is the scatter matrix.

The randomly rough Fresnel boundary modeling is a nontrivial problem as well. There are two approaches to solving this problem [Kargin B. A. 2000]. In the first one, the random field realizations are constructed according to the randomization principle. Then the random trajectories are simulated for the obtained realizations and on their basis estimations for functionals are calculated. However, this requires to find the trajectory and surface intersection points and thus, much computational time.

Therefore, the second approach based on the method of mathematical expectations is preferable. For the construction of a random $N$-trajectory here, realizations of surface elevations are constructed only at $N$-points computed in a certain way. At the points of photon scattering on the random surface, the selection is made random realizations of normals to the surface.

\section{CONCLUSION}

It is obvious now that consideration of the light polarization leads to significant changes in the quantitative results of the calculation of multiple reflections. Moreover, the proposed mathematical model remains within the framework of the standard photometrical concepts but generalizes them to the polarization case. The luminance is a vector value and reflection coefficient transforms into a matrix. The reference plane rotation should be also accounted.
The global illumination equation was generalized on the polarization case and brought to the volume integration as well to mathematically rigorously formulate calculation algorithms with polarization accounting.

Another result of the study is the introduction of light polarization in the algorithm based on the local estimations. This method allows estimating illuminance at the specific points of interest and reduces the calculation time by $80-90$ times.

We are also confident that further development of the model by using the reflecting surface representation described above will enable obtaining more precise results of the light distribution simulation.

\section{ACKNOWLEDGMENTS}

We want to thank the ILEC BL Group and personally its president Boos G. V. for help they provide to us.

\section{REFERENCES}

[Budak et al. 2012] Budak, V.P., Zheltov, V.S., Kalatutsky, T.K. Local estimations of Monte Carlo method with the object spectral representation in the solution of global illumination. Computer Research and Modeling. 2012, Vol. 4, N.1, p.75-84, 2012

[Kajiya J. T. 1986] Kajiya, J.T. The rendering equation. In Proceedings of SIGGRAPH 1986. V.20, N4. - P.143-150, 1986

[Kalos M. 1963] Kalos, M.H. On the Estimation of Flux at a Point by Monte Carlo. Nuclear Science and Engineering. 1963, Vol. 16, N.1, p.111-117, 1963.

[Kargin B. A. 2000] Kargin B.A. Statistical modeling of stochastic problems of the atmosphere and ocean optics. Proc. SPIE 4341, Seventh International Symposium on Atmospheric and Ocean Optics, (29 December 2000).

[Marchuk G. I. 1980] Marchuk, G.I. Monte-Carlo Methods in Atmospheric Optics. Berlin: SpringerVerlag, 1980.

[Mishchenko et al. 1994] Mishchenko, M.I., Lacis, A.A., Travis, L.D. Errors induces be the neglect of polarization in radiance calculations for Rayleigh-scattering atmospheres. Journal of Quantitative Spectroscopy and Radiative Transfer. 1994, Vol. 51, No. 3, pp. 491-510, 1994

[Mojzik et al. 2016] Mojzik, M., Skrivan, T., Wilkie, A., Krivanek, J. Bi-Directional Polarised Light Transport. Eurographics Symposium on Rendering 2016.

[Mueller H. 1943] Mueller, H. Memorandum on the Polarization Optics of the Photo-Elastic Shutter. Report Number 2 of the OSRD Project OEMsr, Boston, MA, USA, 1943; p. 57 